\title{
Control of the Nano-Particle Weight Ratio in Stainless Steel Micro and Nano Powders by Radio Frequency Plasma Treatment
}

\author{
Dong-Yeol Yang ${ }^{1}$, Youngja Kim ${ }^{1}$, Min Young Hur ${ }^{2}$, Hae June Lee ${ }^{2}$, Yong-Jin Kim ${ }^{1}$, \\ Tae-Soo Lim ${ }^{1}$, Ki-Bong Kim ${ }^{1}$ and Sangsun Yang ${ }^{1, *}$
}

1 Powder \& Ceramics Division, Korea Institute of Materials Science, Changwon 642-831, Korea; E-Mails: dyyang@ kims.re.kr (D.-Y.Y.); kimyj12@kims.re.kr (Y.K.); yjkim@kims.re.kr (Y.-J.K.); 1ts6122@kims.re.kr (T.-S.L.); cyrano123@kims.re.kr (K.-B.K.)

2 Department of Electrical Engineering, Pusan National University, Busan 609-735, Korea; E-Mails: myhur@pusan.ac.kr (M.Y.H.); haejune@pusan.ac.kr (H.J.L.)

* Author to whom correspondence should be addressed; E-Mail: nanoyang@kims.re.kr; Tel.: +82-55-280-3517; Fax: +82-55-280-3289.

Academic Editors: Hideshi Miura and Seong Jin Park

Received: 14 October 2015 / Accepted: 2 November 2015 / Published: 6 November 2015

\begin{abstract}
This study describes how to make stainless steel hybrid micro-nano-powders (a mixture of micro-powder and nano-powder) using an in situ one-step process via radio frequency $(\mathrm{RF})$ thermal plasma treatment. Nano-particles attached to micro-powders were successfully prepared by RF thermal plasma treatment of stainless steel powder with an average size of $35 \mu \mathrm{m}$. The ratio of nano-powders is estimated with a two-dimensional fluid simulation that calculates the temperature profile influencing the rate of surface evaporation. The simulation is conducted to determine the variation of the input power and the distance from the plasma torch to the feeding nozzle. It was demonstrated experimentally that the nano-powder ratio in the micro-nano-powder mixture can be controlled by adjusting the feeding rate, plasma power, feeding position and quenching effect during plasma treatment. The ratio of nano-particles in the micro-nano-powder mixture was controlled in a range from 0.1 (wt. \%) to 30.7 (wt. \%).
\end{abstract}

Keywords: metal powder; micro-/nano-powder; RF plasma treatment; metal injection molding; microelectronics; stainless steel 


\section{Introduction}

Components for microelectronics are mostly fabricated by powder metallurgy (PM) processes, such as metal injection molding (MIM), hot isostatic pressing (HIP) and three-dimensional printing (3DP), because of their complicated shapes and small sizes [1-7]. In the past, micro-sized metal-powders were mainly used for these PM processes [8]. However, as component size is gradually decreasing, micro-powder can no longer satisfy the requirements of dimensional accuracy or surface roughness [8,9]. Thus, investigations of MIM, HIP and sintering processes with nano-powder have been actively performed [10-13]. However, if only nano-powder is used, the particle size is very small, and the surface area is large. It is then very difficult to control the extent of oxidation of the powder and to prepare samples with full density. Furthermore, the high price of metal nano-powder, which is related to the productivity of the finished components, is also a problem.

Recent investigations have reported that the enhancement of MIM characteristics and the use of a bimodal-type powder mixture, including a micro-nano-powder, are closely related in $\mathrm{Cu}, \mathrm{Fe}$ and $\mathrm{W}$ systems [10-13]. If MIM products are prepared from mixtures of micro- and nano-powders, molding properties can be improved due to the solid binder effect of the nano-particles; the sintered density will increase due to a nano-powder effect located among the micro-powders, and the grain growth will also be suppressed [10-12]. Surface roughness, an important characteristic of MIM products, can also be improved, and ideally, isostatic shrinkage will be realized $[11,13]$.

As radio frequency (RF) plasma treatment is performed at a very high temperature $\left(>10,000{ }^{\circ} \mathrm{C}\right)$, it is a suitable method to prepare nano-sized metal/ceramic powders [14-18]. RF plasma treatment has been used for the synthesis of inorganic nano-powders or the modification of particle shape (spheroidization); however, in the present study, we sought to make a mixture of micro- and nano-powder using this process. Micro-nano-mixed stainless steel (316L) powders can be prepared by in situ RF thermal plasma treatment, and passivation and atmosphere control in the reaction chamber can prevent severe oxidation of nano-powders.

In order to predict the experimental results, a two-dimensional fluid simulation was conducted for an RF plasma torch. The important features in the thermal plasma synthesis are the heat transfer between the plasma and the precursor powders and the residence time in the plasma region, which influence the rate of the surface evaporation for the production of nano-particles. Therefore, the plasma temperature and velocity fields are important parameters for the synthesis of nano-powders to control the nano-particle ratio and the size distribution. The simulation code is DCPTUN [19,20], which was developed at Seoul National University. This is a single fluid method with magneto-hydro-dynamics (MHD) that is generally used in thermal plasma simulation. The simulation is conducted to determine the variation of the power and the distance from the plasma torch to the feeding nozzle with a fixed gas flow rate and frequency at atmospheric pressure.

The purpose of this study is to make stainless steel hybrid micro-nano-powders using RF plasma treatment and to control the nano-powder ratio by adjusting the process parameters. It is expected that these hybrid micro-nano-powders will be widely used in the powder metallurgy industry, including MIM, HIP and 3DP processes. As this study was performed using a metal model system of stainless steel, the results can be applied to various metal alloys. Furthermore, in this study, we examined the influence of various process parameters (feeding rate, distance from plasma torch to feeding nozzle, 
plasma powder and quenching effect) on the variation of the nano-powder ratio in micro-nano-mixed powders.

\section{Methods}

\subsection{Simulation Methods}

The electromagnetic field generated by a coil current and plasma is calculated using Maxwell's equation of the magnetic vector potential. As the coils are assumed to be parallel, only the azimuthal component of the magnetic vector potential is considered. The governing equation for the magnetic vector potential $\mathbf{A}$ is:

$$
\nabla^{2} \mathbf{A}=-\mu_{0}\left(\mathbf{J}_{\text {coil }}+\mathbf{J}_{\text {induced }}\right)
$$

where $\mu_{0}$ is the permeability of a vacuum and $\mathbf{J}_{\text {coil }}$ and $\mathbf{J}_{\text {induced }}$ are the current density of the coil in the coil region and the induced current density of the plasma in the plasma region, respectively. The boundary condition of $\mathbf{A}$ near the coil is used for the analytic solution of a circular current loop [21,22]. Based on the analytic solution, $\mathbf{A}$ is equal to zero both at the axis boundary and at the boundary far from the coil [22].

Local thermalized equilibrium (LTE) is assumed for the RF plasma torch, which means that the electron and the ion temperatures in the plasma are the same. Thus, the single fluid equation can be used in this case. The MHD equations are used for the conservation of mass, momentum and energy. Moreover, there are several assumptions in this simulation.

(a) Axisymmetric at the cylindrical coordinate for 2D modeling.

(b) Steady-state plasma flow.

(c) The plasma is considered to be an incompressible fluid.

(d) The pressure and viscous dissipation work are neglected in the conservation of energy.

(e) The $K-\in$ model of turbulence is adopted [23].

The governing equations are as below.

(a) Conservation of mass:

$$
\nabla \cdot \vec{u}=0
$$

(b) Conservation of momentum:

$$
\rho \frac{D \vec{u}}{D t}=-\nabla p+\nabla \cdot \vec{\tau}+\overrightarrow{\mathbf{J}} \times \vec{B}
$$

(c) Conservation of energy:

$$
\rho \frac{D h}{D t}=\nabla \cdot\left(k \nabla \frac{h}{C_{p}}\right)+\overrightarrow{\mathbf{J}} \cdot \vec{E}-S_{R}
$$

$\vec{u}, \rho, p, \vec{\tau}, \overrightarrow{\mathbf{J}}, \vec{B}, h, k, C_{p}, \vec{E}$ and $S_{R}$ are the flow velocity, density, pressure, stress tensor, current density, magnetic flux density, enthalpy, thermal conductivity, specific heat, electric field and radiation loss, respectively. 


\subsection{Experimental Methods}

316L stainless steel powders, which are widely used in MIM, exhibit excellent mechanical and corrosion properties, as well as biocompatibility. On this basis, 316L stainless steel was chosen as a model system for this study. For plasma treatment, spherical stainless steel powders $\left(D_{10}=26 \mu \mathrm{m}\right.$, $D_{50}=35 \mu \mathrm{m}, D_{90}=44 \mu \mathrm{m}$, Gaussian distribution) produced using a gas atomization method were used as precursor materials. Production of micro- and nano-mixed powders was carried out with an RF thermal plasma system (PL-35LS, Tekna, Sherbrooke, QC, Canada). The plasma system was operated at $3 \mathrm{MHz}$. Ar gas, vaporized from liquid Ar, was used as a plasma forming gas and carrier gas. The experimental conditions for the plasma treatment are listed in Table 1. Figure 1 shows a schematic diagram of the RF thermal plasma system. Interestingly, the feeding position of the precursor powder can be adjusted up to about $20 \mathrm{~cm}$ below the center of the plasma torch. The powder feeding position can be controlled by changing the length of the feeding probes, and it is possible to inject the precursor powders into the desired temperature regions. Furthermore, installation of 12 quenching gas ports in the reaction chamber affords control over the temperature of the plasma region. Helium (He) was used as the quenching gas. If the $\mathrm{He}$ gas exits at a high flow rate (flow rate $(\mathrm{He})=200-600$ liters per minute (Lpm)) from the quenching gas ports, the plasma region cools down with the heat absorption of helium gas. Exploiting the gas cooling effect, it is possible to control the temperature in the reaction chamber or plasma region. The feeding rate of precursor powders was $10-80 \mathrm{~g} / \mathrm{min}$. To prevent severe oxidation of the nano-powders, a passivation process was conducted.

Table 1. Experimental variables for the preparation of stainless steel 316L hybrid micro-nano-powders using RF plasma treatment.

\begin{tabular}{cc}
\hline Experimental parameter & Value \\
\hline Central gas for plasma (L/min) & $15(\mathrm{Ar})$ \\
Sheath gas for plasma (L/min) & $95(\mathrm{Ar})$ \\
Precursor carrier gas (L/min) & $5(\mathrm{Ar})$ \\
Quenching gas $(\mathrm{L} / \mathrm{min})$ & $200(\mathrm{He})$ \\
Passivation gas $(\mathrm{L} / \mathrm{min})$ & $0.5\left(\mathrm{O}_{2}\right)$ \\
Feeding rate $(\mathrm{g} / \mathrm{min})$ & $10-80$ \\
Chamber pressure $(\mathrm{MPa})$ & 0.1013 \\
Plate voltage $(\mathrm{kV})$ & $5.32-7.16$ \\
Plate current $(\mathrm{A})$ & $2.83-4.22$ \\
Power $(\mathrm{kW})$ & $15-30$ \\
Distance from plasma torch to feeding nozzle $(\mathrm{cm})$ & $0-6.5$ \\
\hline
\end{tabular}




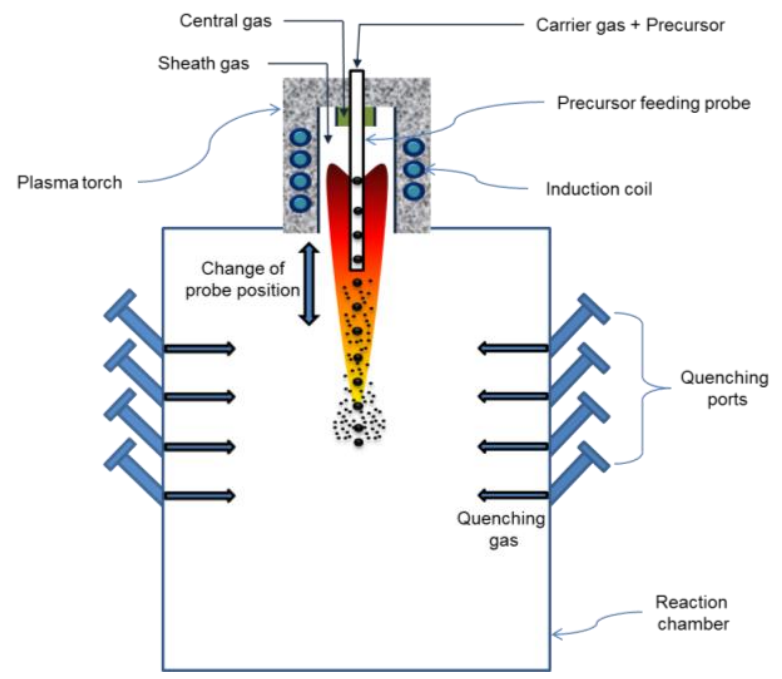

Figure 1. Schematic illustration of precursor feeding probe and quenching ports in the RF thermal plasma system.

The size of the powders was measured using the laser light scattering method (LS13320 MW, Beckman Coulter, Brea, CA, USA); powders with diameter smaller than $100 \mathrm{~nm}$ were defined as nano-powders. The ratio of the nano-powders was determined by measuring the weight of the powder passed through a cyclone trap. The crystal structure of the powders was analyzed using an X-ray diffractometer with $\mathrm{Cu} \mathrm{K}_{\alpha}$ radiation (XRD; D-Max 2200, RIGAKU, Tokyo, Japan). The microstructures of the powders were observed by scanning electron microscopy (SEM; JSM-5800, JEOL, Tokyo, Japan), and the contents of alloy elements were analyzed using inductively-coupled plasma atomic emission spectroscopy (ICP-AES; Optima 5300 DV, Perkin Elmer, Waltham, MA, USA).

\section{Results and Discussion}

Figure 2 shows the simulation results of the temperature profile along the axis line for the variation of the distance from the plasma torch to the feeding nozzle fixed power of $28 \mathrm{~kW}$ in Figure $2 \mathrm{a}$ and for the variation of input power fixed at $0 \mathrm{~cm}$ of the nozzle distance in Figure 2b. The flow rates of argon gas at the central, sheath and carrier regions are 15, 95 and 5 standard liters per minute (sLpm), respectively, as can be seen in Table 1 . The frequency of the RF plasma system is $3 \mathrm{MHz}$ in the simulation, and no quenching gas is applied.

If the distance from the plasma torch to the feeding nozzle increases, the plasma temperature decreases (Figure 2a) because the nozzle is far from the high temperature region near the coil. The temperature influences the surface evaporation of the precursor powders, which means that the high temperature leads to a high ratio of nano-powders. Therefore, we expect a high ratio of nano-powders when there is a short distance between the plasma torch and the feeding nozzle. In Figure $2 b$, it can be seen that with a power increase, the temperature also increases. The ratio of the nano-powders is expected to increase at high power. Additionally, the temperature profiles for a nozzle distance of $2 \mathrm{~cm}$, shown in Figure 2a, and the temperature profile for the case in which the power is $20 \mathrm{~kW}$, shown 
in Figure 2b, are similar. Therefore, the experimental results of for these two temperature profiles are expected to be similar.
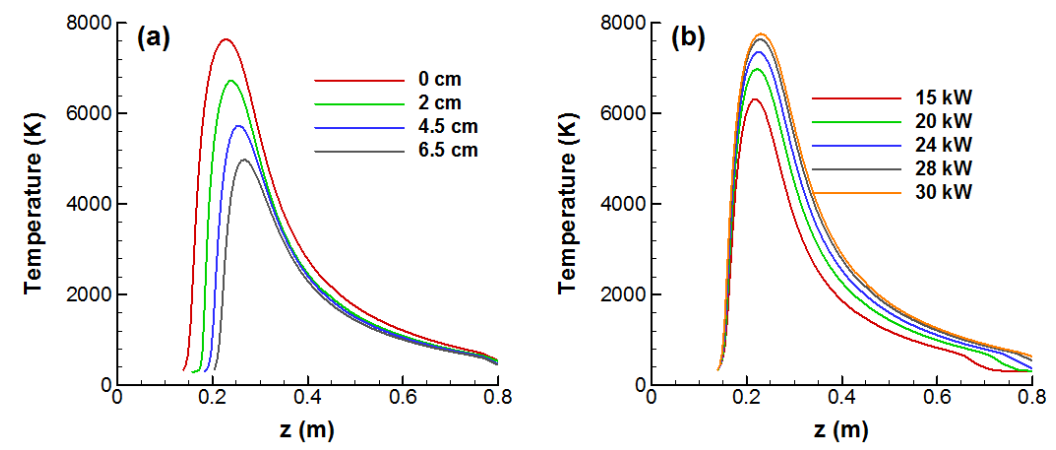

Figure 2. Simulation results showing the temperature profile along the axis line with the variation of (a) the distance from the plasma torch to the feeding nozzle and (b) the plasma power.

As can be seen in Figure 3a,b, the size of the precursor stainless steel powder prepared for plasma treatment was about $D_{50}=35 \mu \mathrm{m}$, and the particle shape was spherical. When RF plasma treatment of this precursor powder was conducted under certain experimental conditions (feeding rate: $10 \mathrm{~g} / \mathrm{min}$; plasma power: $28 \mathrm{~kW}$; distance from the plasma torch to the feeding nozzle: $0 \mathrm{~cm}$ ), hybrid micro-nano-powders consisting of nano-particles (below $100 \mathrm{~nm}$ ) adhering to the surface of the micro-powders, as can be seen in Figure 3c,d, were successfully prepared. The residence time of the precursor powders in a low vacuum plasma state is very short, on the order of microseconds ( $\mu \mathrm{s})$, but the temperature of the reaction zone is more than $10,000{ }^{\circ} \mathrm{C}$. Thus, the stainless steel micro-powder passes through the plasma region, and surface evaporation occurs. Then, as the evaporated particles are again condensed, nano-powders are formed. The micro-sized powders may decrease slightly in size as a result of surface evaporation. Nano-particles adhering to the surface of micro-particles were ultra-fine particles having a size of less than about $100 \mathrm{~nm}$.
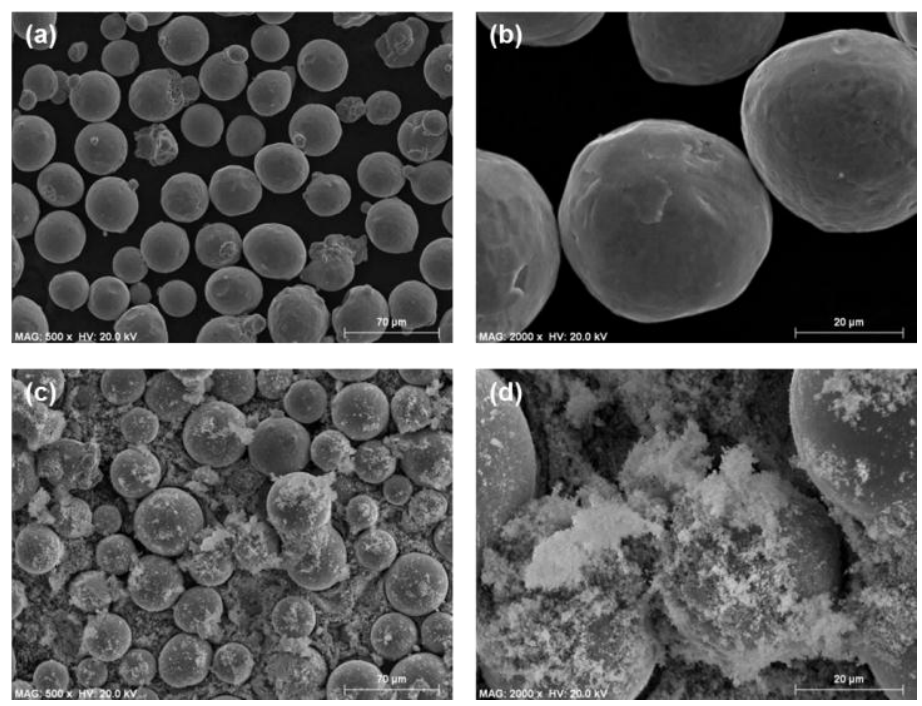

Figure 3. SEM images of the stainless steel 316L powders (a) before and (c) after RF plasma treatment; (b,d) their magnified images. 
To assess the phase transition before and after plasma treatment, XRD analysis was performed. As can be seen in Figure 4, the XRD patterns of the precursor powder and the plasma treated powders are very similar. It is thus surmised that the unique composition of stainless steel (316L) is maintained after the RF plasma treatment. However, the melting point, vaporization temperature and vapor pressure of the alloy elements are different, and the composition ratio of each element after plasma treatment may differ slightly. As can be seen in the results of the ICP-AES analysis before and after plasma treatment (Table 2), the composition ratios of the elements ( $\mathrm{Ni}, \mathrm{Cr}$, Mo) show slight differences in the case of nano-particles, but are all within the composition range of stainless steel $316 \mathrm{~L}$ in the micro-nano-hybrid powders.

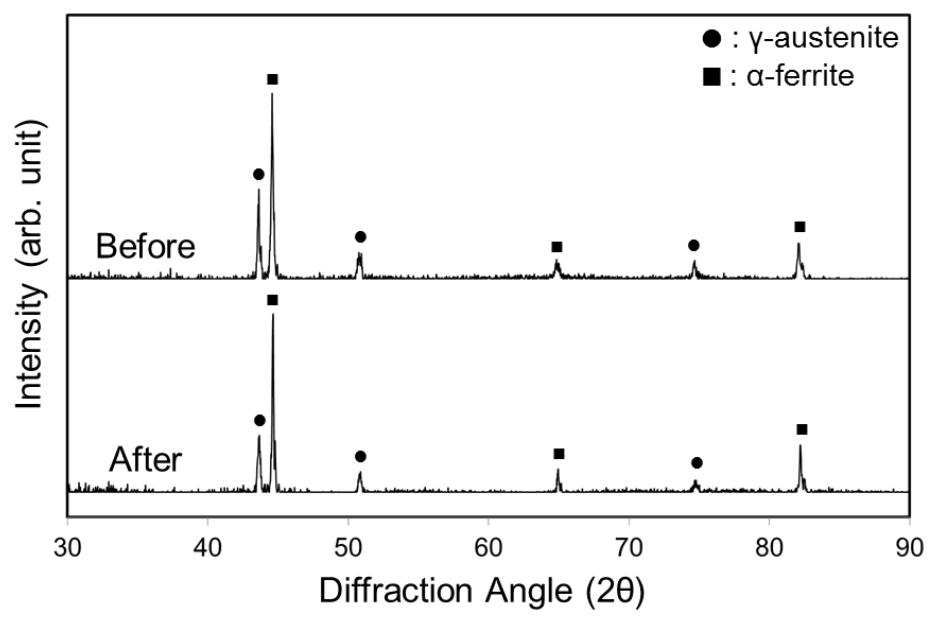

Figure 4. XRD patterns of the powders before and after treatment using RF plasma.

Table 2. Results of the ICP-AES analysis of the powders before and after treatment using RF plasma.

\begin{tabular}{cccccc}
\hline & \multicolumn{5}{c}{ Composition (wt. \%) } \\
\cline { 2 - 6 } Elements & $\begin{array}{c}\text { Reference } \\
\text { (Stainless Steel }\end{array}$ & Atomization & \multicolumn{3}{c}{ Plasma Treated } \\
\cline { 3 - 6 } & 316L) & Precursor & Micro Powder & Nano Powder & $\begin{array}{c}\text { Mixture } \\
\text { (Micro + Nano) }\end{array}$ \\
\hline $\mathrm{C}$ & Max. 0.03 & 0.023 & 0.013 & 0.029 & 0.015 \\
$\mathrm{Si}$ & Max. 0.75 & 0.46 & 0.48 & 0.38 & 0.48 \\
$\mathrm{Mn}$ & Max. 2.00 & 1.18 & 0.67 & 1.95 & 1.05 \\
$\mathrm{P}$ & Max. 0.045 & 0.037 & 0.028 & 0.025 & 0.032 \\
$\mathrm{~S}$ & Max. 0.03 & 0.013 & 0.009 & 0.023 & 0.011 \\
$\mathrm{Ni}$ & $10-14$ & 10.28 & 12.12 & 9.05 & 10.5 \\
$\mathrm{Cr}$ & $16-18$ & 16.94 & 16.43 & 19.06 & 16.6 \\
$\mathrm{Mo}$ & $2-3$ & 2.03 & 2.68 & 0.65 & 2.28 \\
$\mathrm{Fe}$ & Balance & Balance & Balance & Balance & Balance \\
\hline
\end{tabular}

The effects of the experimental parameters on the nano-powder ratio during plasma treatment were examined. First, when the feeding rate was increased from 10 to $80 \mathrm{~g} / \mathrm{min}$, the weight percentage of nano-powders decreased from 19.3 to $0.1 \mathrm{wt}$. \% (Figure 5a). If the feeding rate is increased, as the heat absorbed by particles decreases, the evaporation rate at the particle surface will decrease [24,25]. Therefore, the amount of nano-particles formed by the evaporation-condensation reaction will 
relatively decrease [24,25]. From another viewpoint, if the feeding rate is higher, the amount of non-plasma-treated powder will, in all probability, increase. It is thus reasonable that the amount of nano-powder decreases [26]. Therefore, to increase the nano-powder ratio, the feeding rate should be reduced.
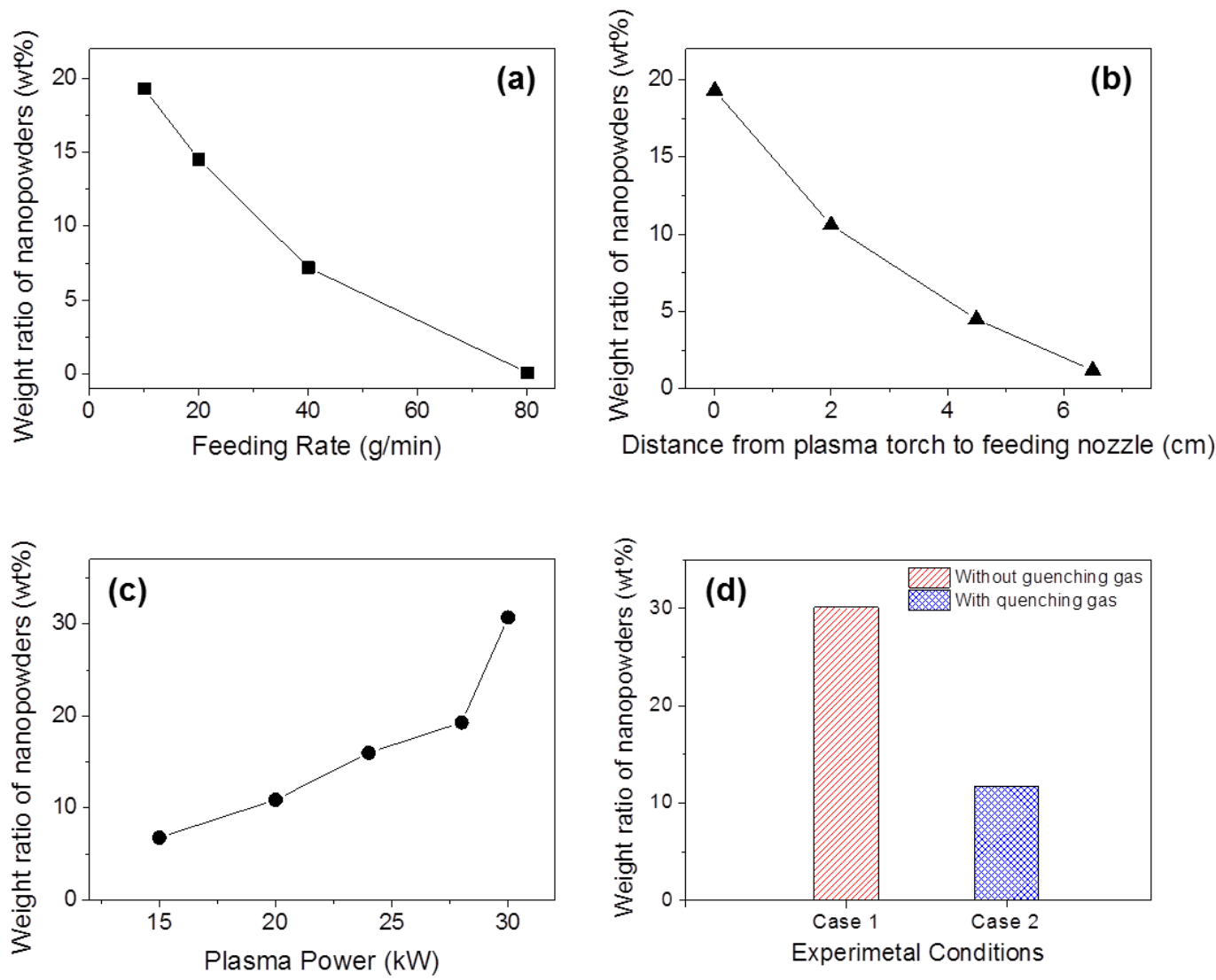

Figure 5. Weight ratio of stainless steel 316L nano-powders with respect to various experimental variables, i.e., (a) feeding rate, (b) distance from the plasma torch to the feeding nozzle, (c) plasma powder and (d) quenching effect, after RF plasma treatment.

To examine the relationship between the nano-powder ratio and the feeding position of the precursor powders, the distance from the center of the plasma torch to the feeding nozzle was increased. As can be seen in Figure 5b, a decrease of the nano-powder ratio occurred as the distance between the plasma torch and the feeding nozzle increased. The reason for this is that the powders can be injected into the higher temperature plasma region when the feeding nozzle is situated closer to the plasma torch. On the contrary, if the feeding nozzle is located farther from the plasma torch, powder will be injected into the low temperature region near the plasma tail; the surface evaporation rate will decrease; and the nano-powder ratio will also decrease.

As the plasma power is increased from 15 to $30 \mathrm{~kW}$, the ratio of nano-powder increases from 6.8 to 30.7 wt. \% (Figure 5c). If the plasma power increases and the temperature of the plasma region increases, the rate of surface evaporation significantly increases, and the nano-powder ratio increases. These results were also predicted by the simulation shown in Figure $2 b$. In other words, as the plasma power increases, the amount of non-plasma-treated powder decreases, and thus, the surface evaporation reaction actively occurs. As can be seen in Figure 1, the helium (He) gas flows into 
12 quenching ports, and the plasma region in the reaction chamber can cool down; that is, it is possible to control the temperature range in the plasma region. If the temperature of the plasma region is decreased due to the release of $\mathrm{He}$ gas (flow rate $(\mathrm{He})=200 \mathrm{~L} / \mathrm{min}$ ), a decrease of the nano-powder ratio (Figure 5d) occurs. If the precursor powders are provided in the low temperature plasma region, the evaporation ratio will decrease, and thus, the nano-powder ratio will decrease. Although the formation ratio of the nano-powders was substantially affected by the four experimental parameters, the effect on the size and morphology of the nano-powders was insignificant. The size of the nano-powders was mostly lower than about $100 \mathrm{~nm}$, and the nano-powders showed spherical shapes.

The influence of the distance from the plasma torch to the feeding nozzle on the nano-powder ratio can be identified in the microstructure. If the feeding position of the precursor powder is moved closer to the plasma torch, the temperature of the plasma region increases. Accordingly, evaporation occurs actively on the surface of the micro-powder exposed to high temperature, and the formation ratio of the nano-powder increases. As can be seen in Figure 6, as the position of the feeding nozzle approaches the center of the plasma torch, the amount of nano-powder $(<100 \mathrm{~nm})$ in the micro-powder increases. These results demonstrate that the nano-powder ratio in the hybrid micro-nano-powder mixture can be controlled by adjusting the process parameters; the microstructural evolutions shown in Figure 6 are experimental evidence of this.
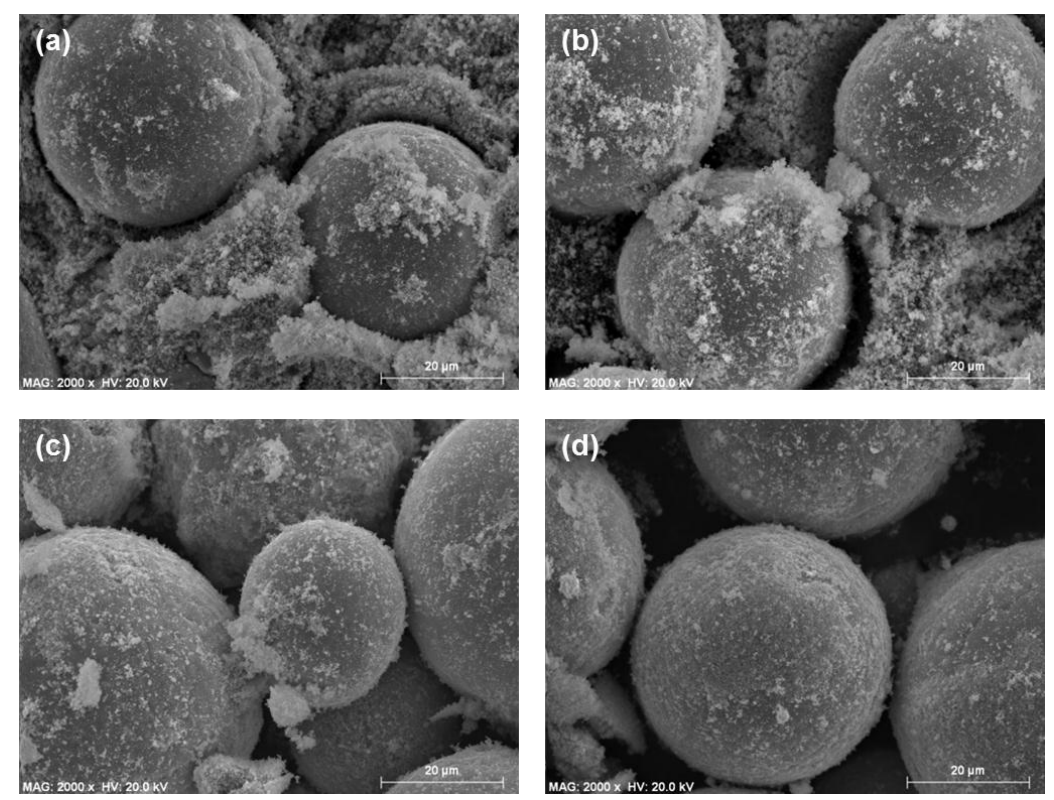

Figure 6. SEM images of the RF plasma-treated stainless steel 316L powders when the distance from the plasma torch to the feeding nozzle is (a) 0 , (b) $2,(\mathbf{c}) 4.5$ and (d) $6.5 \mathrm{~cm}$.

\section{Conclusions}

A novel process to prepare a stainless steel micro- and nano-powder mixture using in situ RF plasma treatment was introduced. The composition of the plasma-treated powders was almost identical to that of the precursor powders. The effect of the process parameters, such as the feeding rate, distance from the plasma torch to the feeding nozzle, plasma powder and quenching effect on the nano-powder ratio, was examined. Before the experiment, the nano-powder ratio is estimated by the calculated temperature field using a two-dimensional single fluid simulation for the variation of the 
plasma power and the distance from the plasma torch to the feeding nozzle. A high ratio of nano-particles is expected in the high temperature region; such a result was obtained using a high power and a short distance between the plasma torch and the feeding nozzle. By adjusting these process parameters, it was possible to control the nano-powder ratio in a range from 0.1 (wt. \%) to 30.7 (wt. \%). The feeding position and the quenching effect are dominant factors that significantly affected the formation ratio of nano-particles. It is anticipated that it will be possible to apply the present findings to the preparation of hybrid micro-nano-powders in other alloy systems.

\section{Acknowledgements}

We would like to acknowledge the financial support from the R\&D Convergence Program of MSIP (Ministry of Science, ICT and Future Planning) and NST (National Research Council of Science and Technology) of the Republic of Korea (Grant B551179-12-02-00). This work was also supported by the Electronics and Telecommunications Research Institute (ETRI) and Civil-Military Technology Cooperation Program (14-BR-MA-05).

\section{Author Contributions}

D.-Y.Y. wrote and edited the paper and contributed to all activities. Y.K. performed SEM and ICP experiment and analyzed the results. M.Y.H. and H.J.L. conducted two-dimensional fluid simulations. T.-S.L. and K.-B.K. synthesized micro-/nano-stainless steel powders using the gas atomizer and RF plasma system. Y.-J.K. and S.Y. contributed to the interpretation and discussion of the experimental results.

\section{Conflicts of Interest}

The authors declare no conflict of interest.

\section{References}

1. Li, Y.; Li, L.; Khalil, K.A. Effect of powder loading on metal injection molding stainless steels. J. Mater. Process. Technol. 2007, 183, 432-439.

2. Ryan, G.E.; Pandit, A.S.; Apatsidis, D.P. Porous titanium scaffolds fabricated using a rapid prototyping and powder metallurgy technique. Biomaterials 2008, 29, 3625-3635.

3. Utela, B.; Storti, D.; Anderson, R.; Ganter, M. A review of process development steps for new material systems in three dimensional printing (3DP). J. Manuf. Process. 2008, 10, 96-104.

4. Ye, H.; Liu, X.Y.; Hong, H. Fabrication of metal matrix composites by metal injection molding. J. Mater. Process. Technol. 2008, 200, 12-24.

5. Contreras, J.M.; Jimenez-Morales, A.; Torralba, J.M. Fabrication of bronze components by metal injection moulding using powders with different particle characteristics. J. Mater. Process. Technol. 2009, 209, 5618-5625.

6. Luo, T.G.; Qu, X.H.; Qin, M.L.; Ouyang, M.L. Dimension precision of metal injection molded pure tungsten. Int. J. Refract. Met. Hard Mater. 2009, 27, 615-620. 
7. Ahsan, M.N.; Pinkerton, A.J.; Moat, R.J.; Shackleton, J. A comparative study of laser direct metal deposition characteristics using gas and plasma-atomized Ti-6Al-4V powders. Mater. Sci. Eng. A 2011, 528, 7648-7657.

8. German, R.M. Powder Injection Molding; Metal Powder Industries Federation: Princeton, NJ, USA, 1990.

9. German, R.M. Powder Metallurgy \& Particulate Materials Processing; Metal Powder Industries Federation: Princeton, NJ, USA, 2005.

10. Nishiyabu, K.; Kakishita, K.; Tanaka, S. Micro Metal Injection Molding Using Hybrid Micro/Nano Powders. Mater. Sci. Forum 2007, 534-536, 381-384.

11. You, W.-K.; Choi, J.-P.; Yoon, S.-M.; Lee, J.-S. Low temperature powder injection molding of iron micro-nano powder mixture. Powder Technol. 2012, 228, 199-205.

12. Han, C.; Choi, H.; Kim, B. Nano-Attached Tungsten Particle Synthesis and Sintering Behaviors. Met. Mater. Int. 2013, 19, 1035-1039.

13. Choi, J.-P.; Lyu, H.-G.; Lee, W.-S.; Lee, J.-S. Densification and microstructural development during sintering of powder injection molded Fe micro-nanopowder. Powder Technol. 2014, 253, 596-601.

14. Károly, Z.; Szépvölgyi, J. Plasma spheroidization of ceramic particles. Chem. Eng. Process. 2005, 44, 221-224.

15. Yang, C.-W.; Lui, T.-S. Effect of Crystallization on the Bonding Strength and Failures of Plasma-Sprayed Hydroxyapatite. Mater. Trans. 2007, 48, 211-218.

16. Gai, G.; Yang, Y.; Jin, L.; Zou, X.; Wu, Y. Particle shape modification and related property improvements. Powder Technol. 2008, 183, 115-121.

17. Harbec, D.; Gitzhofer, F.; Tagnit-Hamou, A. Induction plasma synthesis of nanometric spheroidized glass powder for use in cementitious materials. Powder Technol. 2011, 214, 356-364.

18. Yang, S.; Gwak, J.-N.; Lim, T.-S.; Kim, Y.-J.; Yun, J.-Y. Preparation of Spherical Titanium Powders from Polygonal Titanium Hydride Powders by Radio Frequency Plasma Treatment. Mater. Trans. 2013, 54, 2313-2316.

19. Park, J.H.; Hong, S.H. Optimization Analysis of an Inductively Coupled Plasma Torch for Material Processing by Using Local Thermal Equilibrium Numerical Models. J. Korean Phys. Soc. 1997, 31, 753-763.

20. Park, J.M.; Hur, M.; Hong, S.H. Numerical analysis on effects of various cathode and nozzle geometries on plasma characteristics of transferred arc torches for waste treatment. In Proceedings of the 28th IEEE International Conference on Plasma Science and 13th International Pulsed Power Conference, Las Vegas, NV, USA, 17-22 June 2001.

21. Jackson, J.D. Classical Electrodynamics, 3rd ed.; Wiley: New York, NY, USA, 1998.

22. Bernardi, D.; Colombo, V.; Ghedini, E.; Mentrelli, A. Comparison of different techniques for the FLUENT-based treatment of the electromagnetic field in inductively coupled plasma torches. Eur. Phys. J. D 2003, 27, 55-72.

23. Hur, M.; Hong, S.H. Comparative analysis of turbulent effects on thermal plasma characteristics inside the plasma torches with rod- and well-type cathodes. J. Phys. D 2002, 35, 1946-1954. 
24. Yoshida, T.; Akashi, K. Preparation of Ultrafine Iron Particles using a RF Plasma. Trans. Jpn. Inst. Met. 1981, 22, 371-378.

25. Kobayashi, N.; Kawakami, Y.; Kamada, K.; Li, J.-G.; Ye, R.; Watanabe, T.; Ishigaki, T. Spherical submicron-size copper powders coagulated from a vapor phase in RF induction thermal plasma. Thin Solid Films 2008, 516, 4402-4406.

26. Shin, J.W.; Miyazoe, H.; Leparoux, M.; Siegmann, S.; Dorier, J.L.; Hollenstein, C. The influence of process parameters on precursor evaporation for alumina nanopowder synthesis in an inductively coupled RF thermal plasma. Plasma Sources Sci. Technol. 2006, 15, 441-449.

(C) 2015 by the authors; licensee MDPI, Basel, Switzerland. This article is an open access article distributed under the terms and conditions of the Creative Commons Attribution license (http://creativecommons.org/licenses/by/4.0/). 